\title{
Terminalidade e Condutas de Final de Vida em Unidades de Terapia Intensiva Pediátrica*
}

\author{
Terminal ill Children and End-of-Life Practices \\ in the Pediatric Intensive Care Units
}

\author{
Patrícia Miranda Lago ${ }^{1}$, Daniel Garros², Jefferson P. Piva ${ }^{3}$
}

\section{RESUMO}

JUSTIFICATIVA E OBJETIVOS: Analisar e discutir os aspectos médicos relacionados a terminalidade e cuidados de final de vida oferecidos a crianças internadas, principalmente, em unidades de terapia intensiva pediátrica (UTIP) no Brasil e em alguns países do hemisfério norte.

CONTEÚDO: Foram selecionados artigos sobre cuidados de final de vida publicados nos últimos 20 anos na PubMed, MedLine e LILACS com ênfase nos estudos sobre morte em UTIP no Brasil, América Latina, Europa e Norte América, usando as palavras-chave: morte, bioética, tratamento intensivo pediátrico, reanimação cardiopulmonar e limitação de suporte de vida.

CONCLUSÕES: Observou-se maior incidência de limitação de suporte vital (LSV 60\%) nas UTIP NorteAmericanas e européias que na América Latina (30\%$40 \%$ ). No Brasil a LSV tem diferentes incidências de

1. Pediatra Intensivista do Hospital de Clinicas de Porto Alegre. Doutora em Pediatria pela PUCRS

2. Professor Adjunto de Pediatria, Stollery Children's Hospital, Dept Critical Care, University of Alberta, Edmonton, AB, Canadá

3. Professor Adjunto dos Departamentos de Pediatria da PUCRS e da UFRGS. Chefe Associado da Unidade de Terapia Intensiva Pediátrica do Hospital São Lucas-PUCRS. Secretário da World Federation of Pediatric Intensive and Critical Care Societies (WFPICCS)

*Recebido do Departamento de Pós-Graduação em Pediatria da Pontifícia Universidade Católica do Rio Grande do Sul (PUCRS), Porto Alegre, RS

Apresentado em 31 de agosto de 2007

Aceito para publicação em 14 de setembro de 2007

Endereço para correspondência:

Dra. Patrícia Miranda Lago

Rua Furriel Luis Vargas 238/201

90470-130 Porto Alegre, RS

Fone/Fax: (51) 3333-4462 - Cel. 9678-0431

E-mail: lagopatricia@terra.com.br

(C)Associação de Medicina Intensiva Brasileira, 2007 acordo com a região, tendo aumentado na última década de $6 \%$ para $40 \%$, sendo a ordem de não reanimar a forma mais freqüente. A participação da família no processo decisório no nosso meio é desestimulada e muito incipiente. Baseados na literatura revisada e em sua experiência os autores apresentam as medidas que consideram mais eficazes e recomendadas no seu manuseio. Apesar da LSV em crianças em fase final de doença irreversível ter amparos ético, moral e legal; essas medidas são ainda adotadas de forma tímida. Entre as mudanças urgentes neste comportamento, destaca-se a necessidade do envolvimento da família no processo decisório.

Unitermos: ética médica, limitação de suporte vital, morte, terapia intensiva pediátrica

\section{SUMMARY}

BACKGROUND AND OBJECTIVES: To analyze and discuss the medical practices related to the end-of-life care provided to children admitted to pediatric intensive care unit (PICU) in Brazil and in some countries located in the northern hemisphere.

CONTENTS: Selected articles on end-of-life care published during the last years searching the PubMed, MedLine and LILACS database, with special interest on studies of death conducted in pediatric intensive care units in Brazil, Latin America, Europe and North America, using the following key words: death, bioethics, PICU, cardiopulmonary resuscitation and life support limitation (LSL).

CONCLUSIONS: In North America and North Europe, the incidence of LSL is greater $(60 \%-80 \%)$ than in south Europe and Latin America (30\%-40\%). In Brazil the incidence of LSL depends on the region and in the last decade it is increasing from $6 \%$ to $40 \%$; being the donot-reanimated order the most frequent mode of LSL. The family participation in the decision making process is not stimulated and incipient. Based on the literatu- 
re review and on their experience the authors present the measures that they consider most efficient and recommended for managing this situation in our region. Despite of LSL in children with terminal and irreversible disease be considered ethically, morally and legally; these measures are still adopted in a very few circumstances in our region. Urgent changes in this behavior are necessary, specially related to family participation in the decision-making process.

Key Words: death, life support limitation, medical ethics, pediatric intensive care

\section{INTRODUÇÃO}

Durante os anos 1980 e 1990 houve um grande aumento no número de unidades de terapia intensiva pediátrica (UTIP) em todo o mundo. Este período foi marcado por progressos tecnológicos e melhora nos cuidados oferecidos a crianças criticamente enfermas ${ }^{1-3}$. Estes avanços permitiram diminuição na mortalidade em UTIP, inclusive em paises em desenvolvimento como o Brasil. A mortalidade nestas unidades que se situava entre $15 \%$ e $20 \%$ reduziu para $3 \%$ a $10 \%{ }^{4-7}$. Observou-se também, uma mudança no perfil dos pacientes que faleciam nestas unidades, já que com a evolução nos cuidados primários da infância, a grande maioria das crianças que morrem em UTIP apresenta alguma comorbidade, semelhante ao observado em unidades que atendem pacientes adultos ${ }^{2}$.

Apesar da morte em UTIP não ser uma surpresa, esse ainda é um assunto pouco discutido na literatura, principalmente em paises da América Latina. Pelo menos dois fatores interligados contribuem para que as pesquisas sobre morte em UTIP sejam em número tão reduzido: a baixa mortalidade nestas unidades e a alta expectativa de cura. Como conseqüência da grande potencialidade de cura das crianças, na maioria das vezes, as preocupações com os cuidados de final de vida e a qualidade da morte por parte da equipe médica, ocorrem tardiamente, quando todas as possibilidades de recuperação já se mostraram esgotadas ${ }^{1,2,8,9}$.

\section{TERMINALIDADE EM UTIP}

Pode-se classificar a morte de crianças admitidas em UTIP em dois grupos:

1) Morte inesperada: como as que se seguem ao trauma ou choque séptico, por exemplo. Nestes casos a morte ocorre mesmo após a utilização de toda terapêutica disponível. Felizmente estes pacientes repre- sentam uma minoria;

2) Morte esperada: como nos casos de tumores inoperáveis, doenças crônicas e presença de falência de múltiplos órgãos, após longos tratamentos que se mostraram ineficazes. Na maioria desses casos, depois de identificada a irreversibilidade da situação, o paciente é considerado terminal e se decide pela limitação terapêutica, permitindo que doença tenha seu curso natural, contra-indicando as manobras de reanimação cardiopulmonar (RCP).

A definição de terminalidade é ampla e complexa. Este conceito baseia-se em dados objetivos (por exemplo, resultado de exames como ressonância magnética, tomografia computadorizada e biopsias), subjetivos (como falta de resposta terapêutica a determinado tratamento) e pessoais (experiências próprias anteriores de cada profissional). Obviamente que a determinação de irreversibilidade será muito mais precisa quando baseada predominantemente em dados objetivos ${ }^{10}$. O momento da definição de irreversibilidade freqüentemente varia entre os profissionais envolvidos no atendimento. Esses diferentes momentos devem ser respeitados, para que todos atinjam um diagnóstico e prognóstico consensual ${ }^{2,10}$.

$\mathrm{Na}$ infância, a definição de terminalidade é mais complexa que no adulto. Além da morte de uma criança representar uma situação trágica, a expectativa de reversão do quadro agudo é sempre o maior objetivo da equipe assistencial levando em consideração a grande capacidade de recuperação dos pacientes pediátricos. Portanto, se pode afirmar que a definição da irreversibilidade na criança é um processo mais árduo e que demanda um tempo mais longo.

As condutas de final de vida em Pediatria visam sempre atender o melhor interesse da criança ${ }^{1,11-13}$. Entretanto, diferentemente dos adultos, na maioria das vezes, a criança não tem capacidade de escolher e participar do processo decisório, por isso o papel da família é fundamental. Associado a isto é importante salientar que a relação das crianças com os membros da família e as suas necessidades psicológicas e espirituais diferem dos adultos influenciam o atendimento no momento da morte $2,3,11,14$.

\section{LIMITAÇÃO DE SUPORTE DE VIDA EM PEDIATRIA}

Os primeiros estudos sobre LSV em crianças foram publicados no inicio dos anos 1990 e demonstraram que mais de $30 \%$ dos óbitos em UTIP americanas eram precedidos por alguma forma de LSV ${ }^{2,15}$. 
Esta preocupação em determinar o limite de investimento no paciente pediátrico tem se mostrado cada vez maior na última década, em todo mundo. Atualmente, dependendo da região avaliada, a incidência de LSV em UTIP varia entre $30 \%$ e $80 \%$ 5,16-19. Vários fatores influem na incidência de LSV e também na forma como estas práticas são aplicadas. Fatores culturais, religiosos e até econômicos foram descritos ${ }^{5,20-22}$. Estudos europeus mostram condutas mais agressivas de LSV no norte da Europa, onde a prática médica é mais semelhante à adotada nos EUA e no Canadá, priorizando a autonomia do paciente. No sul europeu que possui cultura com características latinas, as condutas de LSV são menos freqüentes e se baseiam fundamentalmente no paternalismo médico ${ }^{2,5}$.

A unidade do Stollery Children's Hospital no Canadá comparou os dados sobre LSV de sua primeira publicação ${ }^{23}$ em 1990 com a prática corrente de LSV (2003). Observaram que a retirada de suporte ou ordem de não reanimar aumentou de $49 \%$ para $79 \%$ e, conseqüentemente, significativa redução no número de mortes após tentativa de RCP ( $28,7 \%$ versus $9,3 \%)$, mesmo que a UTIP tenha duplicado sua capacidade e aumentado a complexidade dos serviços, como programa de cirurgia cardíaca corretiva, serviço de transporte regional e ECMO (dados não publicados).

$\mathrm{Na}$ América do Sul existem poucas pesquisas com método e amostragem representativos que tenham estudado práticas de LSV em Pediatria. A Medicina dos paises da América do Sul caracteriza-se por um forte componente paternalista, como no sul da Europa. Associado a isto, o conceito tradicional de que a vida deve ser mantida a qualquer preço ainda permanece enraizado na sociedade civil e na prática médica destes paises. Não existe uma tradição de valorização da autonomia do paciente, e as decisões de final de vida acabam sendo tomadas pela equipe médica baseadas no conceito de beneficência e influenciadas pelos valores culturais e morais de cada equipe de profissionais da saúde².

A maioria dos relatos da América Latina começou a aparecer no início do ano 200024-27. Um estudo argentino de 2003 avaliando o modo de morte de crianças internadas em UTIP de 16 hospitais, demonstrou que $52 \%$ dos pacientes internados recebiam RCP completa antes do óbito e a forma de LSV mais freqüentemente utilizada era a ordem de não reanimar (ONR) ${ }^{28}$.

No Brasil, três estudos muito semelhantes ao da Argentina avaliaram os modos de morte de crianças internadas em UTIP. Kipper e col. publicaram uma pes- quisa envolvendo três UTIP de referência na região sul do Brasil, com intervalo de 10 anos, demonstrando um significativo aumento na limitação de suporte de vida (6\% para $30 \%$ ) entre os anos de 1988 e $1998^{25}$. Em 2005, Lago e col.; Tonelli e col. em estudos simultâneos, descreveram um aumento na adoção de LSV em UTIP das regiões sul e sudeste ${ }^{26,29}$. Estes três estudos demonstraram que o aumento de práticas de LSV ocorreu basicamente à custa de ONR ou seja, em grande parcela de crianças que morrem UTIP brasileiras, o suporte de vida pleno é mantido até que ocorra a parada cardiorrespiratória do paciente.

Mais recentemente, Lago e col. ${ }^{7}$ realizaram nova análise multicêntrica em três regiões diferentes do Brasil, totalizando 428 mortes em sete UTIP, sendo duas em Porto Alegre, duas em São Paulo e três em Salvador, no período de 2003 a 2004. Não houve diferença quanto à idade, sexo, número de órgãos em disfunção e septicemia como maior causa de morte. A RCP ainda antecedeu $57 \%$ das mortes, com significativa diferença entre as regiões sudeste e o nordeste. Ficou evidente nesse estudo que a ONR estava associada ao tempo de internação em UTIP (mais de cinco dias). Este fato também foi observado em UTIP de países desenvolvidos, porém difere do descrito em algumas UTI de adultos, onde pacientes considerados terminais tem tempo de internação menor do que aqueles com doenças agudas 5,30 .

O que surpreende na análise brasileira é que somente quatro dos 186 pacientes que não receberam RCP, os fármacos vasoativos foram descontinuados. Na maioria dos pacientes, esses fármacos foram aumentados e os parâmetros ventilatórios foram ainda incrementados nas últimas $48 \mathrm{~h}$ antes da morte. No entanto, no mesmo estudo, observou-se entre os pacientes com decisão de ONR que em 34\% houve redução na infusão de inotrópicos e em $16 \%$ dos óbitos ocorridos na região sul não foi instituída via aérea artificial ou ventilação mecânica. Estes dados foram descritos pela primeira vez no Brasil e demonstraram mudança positiva em direção a uma prática mais ativa e coerente nos cuidados de final de vida.

Os estudos sobre morte no Brasil e na América do Sul demonstram a dificuldade da equipe médica em aplicar LSV em crianças e que estas condutas variam de região para região. Apesar do aumento da "não oferta" ou "retirada de suporte de vida" nos momentos que antecedem a morte, a ONR ainda é o modo mais freqüente. A maioria dos estudos tenta justificar esta preferência de conduta, diferente da dos paises do he- 
misfério norte, como estando relacionada a características culturais do povo ${ }^{28}$. Porém fica difícil quantificar o quanto é reflexo de tradições religiosas, culturais e legais de países sul-americanos e o quanto é conseqüência do despreparo médico sobre cuidados de final de vida, associado à pequena preocupação em oferecer ao paciente uma morte mais digna.

\section{EXISTE UMA ROTINA PARA APLICAR AS CONDU- TAS DE LSV?}

O processo de LSV pode ser dividido em 4 fases: 1. Inicio da discussão com definição da irreversibilidade dentro da equipe assistencial. A fase de comunicação da irreversibilidade para a família só pode ser iniciada após o convencimento de todos os envolvidos na assistência (médico assistente, intensivista, especialista e equipe de enfermagem);

2. Esclarecimento do prognóstico para a família pelo médico e enfermeira que participaram do atendimento. Esta conversa deve ser feita em um ambiente calmo e repetida quantas vezes forem necessárias. Dificilmente os familiares de um paciente crítico aceitam com facilidade a irreversibilidade da situação e tampouco entenderão se este diálogo ocorrer em um corredor de hospital. Neste momento, o médico deve se comunicar de forma clara, evitar termos técnicos e estimular que a família apresente suas duvidas e fantasias. É importante que a equipe assistencial tenha uma postura de solidariedade e compreensão; assim como se disponha a discutir as alternativas apresentadas pela família;

3. Identificação de metas para o fim da vida. O médico deve oferecer à família as possibilidades terapêuticas que considere adequadas naquele momento e para que juntos decidam pelo melhor interesse do paciente;

4. Desenvolvimento conjunto família-equipe assistencial de um plano de tratamento que deve ter como principal meta o conforto do paciente. A administração de analgésicos e sedativos é uma intervenção terapêutica primordial nos cuidados finais ${ }^{12}$. Se doses supraterapêuticas de morfina forem necessárias para garantir conforto, isto é respaldado pelo princípio ético do duplo efeito. Quando se opta por remover alguma terapêutica, a família deve estar segura de que o tratamento que está sendo retirado é fútil e não alterará a evolução da doença. A morte deste paciente correrá inexoravelmente recebendo ou não esta intervenção.

Do ponto de vista ético não existe um modo de LSV ideal ou mais correto, porém sabe-se que o fundamen- tal é permitir ao paciente uma morte tranqüila e sem sofrimento e aos seus familiares à possibilidade de compartilhar estes últimos momentos em um ambiente de privacidade ${ }^{1}$.

\section{CONCLUSÃO}

Apesar da defasagem tecnológica e dos graves problemas organizacionais, os intensivistas pediátricos brasileiros conseguiram resultados nas últimas décadas que causam orgulho a todos. Talvez, obstinados em reduzir a mortalidade, tenham se descuidado do atendimento de uma pequena parcela de pacientes que falecem nas UTIP em conseqüência de doenças terminais. A obstinação pela cura neste grupo é a antítese do bom atendimento. Estas crianças necessitam de cuidados que visem uma morte em paz. Atender esta demanda mínima não pode configurar algo hierarquicamente menos importante.

Os intensivistas brasileiros sabem que a LSV em pacientes com doença irreversível tem amparo moral, ético, religioso e inclusive legal. Na opinião dos autores, os problemas com a LSV em pacientes terminais internados em UTIP brasileiras estão relacionados a:

I) Dificuldade do grupo médico em obter o consenso quanto à terminalidade;

II) Falta de treinamento e embasamento na arte de "cuidar";

III) Insegurança na definição do momento da terminalidade e dificuldade de comunicação com a família.

Todavia, frente ao crescente número de publicações relacionado a este assunto, assim como a própria Resolução CFM 1.805/06 que deu ampla publicidade a este problema na imprensa leiga, tem-se convicção que se está vivenciando um processo de profundas mudanças no cuidado de crianças com doenças terminais internadas em UTIP no Brasil ${ }^{31}$.

\section{REFERÊNCIAS}

01. Truog RD, Meyer EC, Burns JP - Toward interventions to improve end-oflife care in the pediatric intensive care unit. Crit Care Med, 2006;34:(Suppl11):S373-S379.

02. Lago PM, Devictor D, Piva JP et al - End-of-life care in children: the Brazilian and the international perspectives. J Pediatr, 2007;83:(Suppl2): S109-S116.

03. Garros D, Rosychuk RJ, Cox PN - Circumstances surrounding end of life in a pediatric intensive care unit. Pediatrics, 2003;112:E371-E379.

04. Lago PM, Piva J, Garcia PC et al - Brain death: medical management in seven Brazilian pediatric intensive care units. J Pediatr, 2007;83:133-140.

05. Devictor DJ, Nguyen DT - Forgoing life-sustaining treatments in children: a comparison between Northern and Southern European pediatric intensive care units. Pediatr Crit Care Med, 2004;5:211-215. 
06. Carcillo JA - What's new in pediatric intensive care. Crit Care Med, 2006;34:(Suppl9):S183-S190.

07. Lago PM, Piva J, Garcia PC et al - End of life practices in seven Brazilian Pediatric Intensive Care Units (PICU). Pediatr Crit Care Med, 2007; (accepted for publishing)

08. Mateos C, Lopez PR, Diaz JS et al - Cuidados al final de la vida en la unidad de cuidados intensivos pediátrica. Revisión de la bibliografía. Ann Pediatr, 2006;63:152-159.

09. Bearison DJ - When Treatment Fails: How Medic Cares for Dying Children. Oxford University, 2006;332.

10. Piva JP, Carvalho PR - Considerações éticas nos cuidados médicos do paciente terminal. Bioética, 1993;1:139-144.

11. Garros D - A "good" death in pediatric UCl: is it possible? J Pediatr, 2003;79:(Suppl2):S243-S254.

12. Carlet J, Thijs LG, Antonelli M et al - Challenges in end-of-life care in ICU. Statement of the $5^{\text {th }}$ International Consensus Conference in Critical Care: Brussels, Belgium, April 2003. Intensive Care Med, 2004;30:770-784.

13. Carter BS, Howenstein M, Gilmer MJ et al - Circumstances surrounding the deaths of hospitalized children: opportunities for pediatric palliative care. Pediatrics, 2004;114; e361-e366.

14. Truog RD, Cist AF, Brackett SE et al - Recommendations for end-of-life care em the intensive care unit: The Ethics Committee of the Society of Critical Care Medicine. Crit Care Med, 2001;29:2332-2348.

15. Mink RB, Pollack MM - Resuscitation and withdrawal of therapy in pediatric intensive care unit. Pediatrics, 1992;89:961-963.

16. Wunsch $\mathrm{H}$, Harrison DA, Harvey $\mathrm{S}$ et al - End-of-life decisions: a cohort study of the withdrawal of all active treatment in intensive care units in United Kingdom. Intensive Care Med, 2005;31:823-831.

17. Zawistowski CA, deVita MA - A descriptive study of children dying in the pediatric intensive care unit, after withdrawal of life-sustaining treatment. Pediatr Crit Care Med, 2004;5:216-223.

18. Prendergast TJ, Luce JM - Increasing incidence of withholding and withdrawal of life support from the critically ill. Am J Respir Crit Care, $1997 ; 155: 15-20$.
19. Gajewska K, Schroeder M, De Marre F et al - Analysis of terminal events in 109 successive deaths in a Belgian intensive care unit. Intensive Care Med, 2004;30:1224-1227.

20. Mani RK - End-of-life care in India. Intensive Care Med, 2006;32:10661068.

21. Yaguchi A, Truog RD, Curtis JR et al - International differences in endof-life attitudes in the intensive care unit: results of a survey. Arch Intern Med, 2005;165:1970-1975.

22. McLean RF, Tarshis J, Mazer CD et al - Death in two Canadian intensive care units: institutional differences and changes over time. Crit Care Med, 2000;28:100-103.

23. Ryan CA, Byrne P, Kuhn $S$ et al - No resuscitation and withdrawal of therapy in a neonatal and a pediatric intensive care unit in Canada. $J$ Pediatr, 1993;123:534-538.

24. Carvalho PR, Rocha TS, Santo AE et al - Modes of death in the PICU of a tertiary care hospital. Rev Assoc Med Bras, 2001;47:325-331.

25. Kipper DJ, Piva JP, Garcia PC et al - Evolution of the medical practices and modes of death on pediatric intensive care in southern Brazil. Pediatr Crit Care Med, 2005;6:258-263.

26. Lago PM, Piva J, Kipper D et al - Life support limitation at three pediatric intensive care units in southern Brazil. J Pediatr, 2005;81:111-117.

27. Moritz RD, Pamplona F - Avaliação da recusa ou recusa de tratamentos considerados fúteis ou inúteis em UTI. RBTI, 2003;15:40-44.

28. Althabe M, Cardigni G, Vassallo JC et al - Dying in the intensive care unit: collaborative multicenter study about forgoing life-sustaining treatment in Argentine pediatric intensive care units. Pediatr Crit Care Med, 2003;4:164-169.

29. Tonelli HA, Mota JA, Oliveira JS - A profile of the medical conduct preceding child death at a tertiary hospital. J Pediatr, 2005;81:118-125.

30. Cook D, Rocker G, Marshall J et al - Withdrawal of mechanical ventilation in antecipation of death in the intensive care unit. N England J Med, 2003;349:1123-1132.

31. Conselho Federal de Medicina. Resolução CFM 1.805/2006. Diário Oficial da União 2006;28: 196. 